\title{
DRENAGEM LINFÁTICA MANUAL NO TRATAMENTO DA SÍNDROME PRÉ-MENSTRUAL
}

Francely de Castro e Sousa, Breno Gontijo do Nascimento, Eduardo Alonso. Drenagem linfática manual no tratamento da síndrome pré-menstrual. Revista Saúde Dinâmica, vol. 2, núm.4, 2020. Faculdade Dinâmica do Vale do Piranga.

\section{SAÚDE DINÂMICA - Revista Científica Eletrônica FACULDADE DINÂMICA DO VALE DO PIRANGA}

6a Edição 2020 | Ano II - no 4 | ISSN-2675-133X 


\title{
Drenagem linfática manual no tratamento da síndrome pré-menstrual Manual lymphatic drainage in the treatment of pré-menstrual syndrome
}

\author{
Francely de Castro e Sousa ${ }^{1}$, Breno Gontijo do Nascimento ${ }^{2}$, Eduardo Alonso ${ }^{3}$ \\ ${ }^{I}$ Fisioterapeuta, Docente e Assistente de Coordenação do Curso de Fisioterapia, Faculdade Dinâmica do Vale do Piranga, \\ Ponte Nova, Brasil, ORCID: 0000-0002-1881-6409 \\ ${ }^{2}$ Docente de Fisioterapia, Centro Universitário Estácio, Belo Horizonte, Brasil, ORCID: 0000-0002-0179-3001 \\ ${ }^{3}$ Médico Ginecologista, Docente do Instituto Universitário Italiano de Rosário, Argentina, ORCID: 0000-0002-5339-8775 \\ Autor correspondente: francelycastro@hotmail.com
}

\section{Resumo}

A síndrome pré-menstrual (SPM) é um conjunto de sintomas que ocorrem nos dias que precedem a menstruação. Os sintomas físicos estão relacionados ao edema, que pode ser tratado pela Fisioterapia, por meio da Drenagem Linfática Manual (DLM). Objetivou-se avaliar os efeitos da DLM em mulheres com retenção hídrica no período pré-menstrual. Materiais e Métodos: Estudo do tipo antes e depois com abordagem quantitativa. Foram incluídas 49 voluntárias, que assinaram consentimento informado. $\mathrm{O}$ estudo foi aprovado pelos Comitês de Ética do Brasil e da Argentina. As voluntárias foram avaliadas e acompanhadas por dois ciclos menstruais consecutivos. No primeiro ciclo, como grupo controle, peso, porcentagem de água, medidas de perimetria e sintomas relatados foram aferidos e registrados. No ciclo seguinte, as mesmas voluntárias formaram o grupo intervenção, foram submetidas a sete sessões de DLM, e colhidas as mesmas variáveis. Procedeu-se análise estatística pelo SPSS. Todas as variáveis apresentaram queda final, quando se compara o ciclo de intervenção com o ciclo de controle. A DLM evitou aumento nas variáveis peso, perimetria e porcentagem de água corporal e sintomas pré-menstruais relatados. A técnica deve ser aplicada em todo o corpo.

Palavras-chave: Síndrome Pré-Menstrual; Drenagem linfática manual; Edema; Fisioterapia.

\begin{abstract}
Premenstrual syndrome (PMS) is a set of symptoms that occur in the days preceding menstruation. Physical symptoms are related to edema, which can be treated by Physiotherapy, through Manual Lymphatic Drainage (DLM). The objective of this study was to evaluate the effects of MLD in women with water retention in the premenstrual period. Materials and Methods: Study of the type before and after with quantitative approach. 49 volunteers were included, who signed informed consent. The study was approved by the Ethics Committees of Brazil and Argentina. The volunteers were evaluated and followed up for two consecutive menstrual cycles. In the first cycle, as a control group, weight, water percentage, perimeter measurements and reported symptoms were measured and recorded. In the following cycle, the same volunteers formed the intervention group, were submitted to seven sessions of DLM, and the same variables were collected. Statistical analysis was performed by SPSS. All variables showed a final drop when comparing the intervention cycle with the control cycle. DLM prevented an increase in the variables weight, perimeter and percentage of body water and reported premenstrual symptoms. The technique must be applied throughout the body.
\end{abstract}

Key words: Premenstrual Syndrome; Manual Lymphatic Drainage; Edema; Physiotherapy. 


\section{INTRODUÇÃO}

A síndrome pré-menstrual (SPM) é um conjunto de sintomas que ocorrem durante os dias que precedem a menstruação. Eles são divididos em somáticos e físicos, e podem ser causados por alterações nos níveis de estrogênio e progesterona (CAMPAGNE; CAMPAGNE, 20007).

O ciclo menstrual dura cerca de 28 dias e é dividido em 3 fases: a fase folicular, que são os primeiros 13 dias; a fase de ovulação, que ocorre cerca de 3 dias após a fase folicular; e a fase lútea, que vai do final da ovulação até o segundo dia após a chegada do ciclo menstrual (GUYTON; HALL, 1998).

A fase lútea tardia é a fase com maior evidência de edema abdominal e de membros inferiores, acompanhada de dor abdominal. Isso se deve a uma diminuição nas concentrações dos hormônios estrogênio e progesterona, um desequilíbrio no sistema de Starling que contribui para o aumento da pressão hidrostática com perdas de plasma e íons para o interstício, entre outros (ROSENFELD et al., 2008).

Para Augusto e Urbanetz (1985), o edema é um sintoma pré-menstrual frequente, afetando $92 \%$ das mulheres, predominantemente na fase lútea do ciclo, quando o principal hormônio é a progesterona. Esse hormônio causa flacidez da parede venosa e compromete a drenagem, causando retenção de água.

Em seu trabalho, Gonda et al. (2008) verificaram que mais da metade de um total de 63 mulheres apresentou aumento na retenção de água superior a $66 \%$ em questionário específico para avaliação de sintomas menstruais na fase lútea.

Sintomas físicos, sensação de inchaço, cefaleia, dor no abdômen, entre outros, estão relacionados ao edema, ou seja, a existência de fluidos intersticiais que são decorrentes de uma alteração na drenagem linfática (CAMPAGNE; CAMPAGNE, 20007).

O sistema linfático é responsável pela drenagem de fluidos. Possui vasos linfáticos muito finos que se originam em quase todos os espaços do tecido, conhecidos como capilares ou vasos linfáticos iniciais; eles se fundem gradualmente em grandes vasos linfáticos, que terminam na região do pescoço, onde a linfa é direcionada para a junção das veias jugulares internas com as veias subclávias, nos lados direito e esquerdo. É um sistema acessório que drena macromoléculas de proteínas e fluidos dos espaços intersticiais e envia para os grandes vasos linfáticos, passando pelos linfonodos, que filtram a linfa, promovendo assim a remoção de todas 
as partículas indesejáveis do fluido antes de ser direcionado à rede venosa, o que permite o retorno das proteínas ao sangue, regulando a pressão osmótica vascular (BERNE; LEVY, 2000; GUIRRO; GUIRRO, 2004).

O papel da Fisioterapia é atuar na redução dos sintomas físicos da SPM, incluindo edema, promovendo o equilíbrio homeostático intersticial por meio da Drenagem Linfática Manual (DLM), reduzindo, assim, o desconforto causado pela SPM.

A DLM é uma técnica fisioterapêutica que visa imitar o sistema linfático e reduzir a retenção de água (GUIRRO; GUIRRO, 2004).

Essa técnica ajuda a eliminar os resíduos do metabolismo celular. Os processos que contribuem para a eliminação desses líquidos são as manobras de captação, técnica manual suave, que estimulam as redes capilares. Também estão incluídas as manobras de evacuação, que transportam a linfa que vem da região infiltrada, dos capilares para os vasos pré-coletores, para os vasos coletores (LEDUC; LEDUC, 2007; GODOY; GODOY, 2004).

Para Borges (2006), a DLM aumenta a taxa de filtração da linfa por meio do aumento do fluxo linfático, o volume da corrente linfática pode ser cinco vezes maior, a quantidade de fluido devolvida à circulação aumenta. A DLM descongestiona os tecidos e promove renovação constante do fluido intersticial.

A presença de pouco líquido nos espaços intersticiais é benéfica para a difusão de nutrientes dos capilares para as células. Quando o corpo humano não consegue manter este estado, os espaços intersticiais ficam com excesso de fluido, assim, ocorre a formação de edema e, portanto, há um aumento na distância que os nutrientes devem percorrer para se difundir dos espaços capilares para células de tecido (BERNE; LEVY, 2000).

O edema é um dos sintomas físicos que afeta a maioria das mulheres com SPM. Isso é difícil de diagnosticar porque não existem testes de laboratório que possam identificar biomarcadores. Porém, a avaliação fisioterapêutica pode fornecer um possível diagnóstico por meio da anamnese e da perimetria. O diagnóstico da SPM é feito por ginecologistas que consideram a avaliação de sinais e sintomas, inclusive, psiquiátricos (YONKERS; O’BRIEN; ERIKSSON, 2008).

A retenção de líquidos e seus sintomas causam prejuízos nas atividades da vida diária e na rotina de trabalho das mulheres, existindo poucos os estudos realizados com opções de tratamento não farmacológico. 
Assim, o objetivo geral deste estudo foi avaliar os efeitos da drenagem linfática manual em mulheres com retenção de líquidos pré-menstrual. Os objetivos específicos foram analisar as variáveis peso, porcentagem de água corporal, perimetria e sintomas referidos, em dois ciclos menstruais consecutivos na mesma mulher; verificar as mudanças dessas variáveis nos ciclos de controle e intervenção para demonstrar a eficácia da técnica.

\section{MATERIAIS E MÉTODOS}

Este é um estudo antes e depois. A população do estudo incluiu mulheres da Universidade "Estácio de Sá de Belo Horizonte", alunas do campus Floresta. Este campus universitário tem 1159 mulheres matriculadas em diversos cursos, Enfermagem, Fisioterapia, Direito dentre outros, de acordo com dados da Secretaria Geral de Alunos, em julho de 2013.

Com base no Programa de Análises Epidemiológicas de dados tabulados (EPIDAT), versão 3.1, o tamanho da amostra e a precisão foram calculados para estimar uma proporção da população. A proporção esperada foi considerada com base em Kwan e Onwude (2009) que afirmaram que os sintomas pré-menstruais ocorrem em $95 \%$ das mulheres em idade reprodutiva. O objetivo foi realizar o estudo com um tamanho amostral com precisão de $6 \%$.

Para inclusão das voluntárias foram considerados os seguintes critérios: idade entre 20 e 30 anos, nulíparas, sem abortos prévios, em uso de contraceptivo oral há pelo menos 6 meses, menstruação regular, sedentária e com índice de massa corporal (IMC) entre 18 e 25 kg/m2. Também foi necessário que elas se submetessem a 14 sessões de Fisioterapia, distribuídas em 2 ciclos menstruais consecutivos, sempre no mesmo horário do dia e que pudessem permanecer na sessão por cerca de 1 hora.

Foram excluídas as voluntárias que não apresentavam sintomas de retenção de líquidos ou referissem insuficiência venosa, hipertensão, ou algum processo de disfunção hormonal, inflamatória, infecciosa e / ou tumoral. Também foram excluídas as voluntárias que referiram insuficiência linfática ou com sintomas não relacionados ao período pré-menstrual, ou seja, sintomas que se manifestassem fora da fase lútea do ciclo menstrual ou mesmo que permanecessem além desta fase.

Os seguintes critérios para suspensão foram apresentados no estudo: sensação de dor ou desconforto com a técnica de drenagem linfática manual; necessidade e uso de analgésico e / 
ou diurético; ou ainda, impossibilidade de comparecimento às sessões de Fisioterapia nos dias agendados para coleta de dados ou intervenção.

Uma vez esclarecidos os critérios de inclusão e exclusão, houve disponibilidade de 49 voluntárias, com sintomas de retenção de líquidos no período pré-menstrual, para se submeter a 14 (quatorze) sessões sucessivas, distribuídas na fase lútea de 2 (dois) ciclos menstruais consecutivos. Todas as dúvidas das voluntárias foram esclarecidas e elas assinaram o Termo de Consentimento Livre e Esclarecido.

As voluntárias foram avaliadas e os dados de avaliação devidamente registrados em formulário específico. Elas foram acompanhadas por dois ciclos menstruais.

No primeiro ciclo, foram analisadas como grupo controle, preencheram um diário nutricional, com os horários, quantidades e tipos de alimentos e líquidos consumidos, e um questionário para investigar os sintomas relacionados à percepção do período pré-menstrual.

Foram aferidos e registrados o peso e a porcentagem de água corporal das voluntárias, assim como a circunferência dos braços, mamas, abdômen, quadris, coxas e pernas. Todos os dados coletados foram registrados em formulário próprio. As voluntárias usaram roupa íntima no momento das medições para preservar a privacidade.

No ciclo seguinte, as mesmas voluntárias foram analisadas como grupo de intervenção. Mais uma vez foram avaliadas e, na sequência, submetidas a sete sessões de DLM, de 40 a 60 minutos, em datas pré-determinadas, de acordo com a tabela 1, realizadas por terapeuta treinado, de acordo com a técnica de DLM descrita por Leduc e Leduc (2007).

Os procedimentos de coleta de dados relativos aos sintomas relatados no período prémenstrual, peso, porcentagem de água e medidas da perimetria, foram os mesmos, além disso, as voluntárias foram orientadas a seguir o diário nutricional para realizar a ingestão de alimentos / líquidos de forma semelhante ao ciclo anterior.

Tabela 1: Procedimentos realizados nos dias do ciclo menstrual nos grupos controle (Ciclo I) e intervenção (Ciclo II).

\begin{tabular}{c|ccccccc|ccccccc}
\hline \multicolumn{11}{c}{ Ciclo Controle } & \multicolumn{1}{c}{ Ciclo Intervenção } \\
\hline Dia do Ciclo & $24^{\circ}$ & $25^{\circ}$ & $26^{\circ}$ & $27^{\circ}$ & $28^{\circ}$ & $1^{\circ}$ & $2^{\circ}$ & $24^{\circ}$ & $25^{\circ}$ & $26^{\circ}$ & $27^{\circ}$ & $28^{\circ}$ & $1^{\circ}$ & $2^{\circ}$ \\
Aferições & $\mathrm{X}$ & $\mathrm{X}$ & $\mathrm{X}$ & $\mathrm{X}$ & $\mathrm{X}$ & $\mathrm{X}$ & $\mathrm{X}$ & $\mathrm{X}$ & $\mathrm{X}$ & $\mathrm{X}$ & $\mathrm{X}$ & $\mathrm{X}$ & $\mathrm{X}$ & $\mathrm{X}$ \\
DLM & - & - & - & - & - & - & - & $\mathrm{X}$ & $\mathrm{X}$ & $\mathrm{X}$ & $\mathrm{X}$ & $\mathrm{X}$ & $\mathrm{X}$ & $\mathrm{X}$ \\
\hline
\end{tabular}

Fonte: Elaborado pelos autores. 
No ciclo intervenção, as medidas e a DLM foram realizadas no mesmo horário do dia do ciclo controle e as voluntárias foram orientadas a se vestir da mesma maneira.

Quatro fitas métricas domésticas foram utilizadas, com o cuidado de se utilizar a mesma fita métrica no ciclo de controle e no ciclo intervenção para cada uma das voluntárias.

Duas balanças com bioimpedância, previamente calibradas, uma da marca Tanita modelo AC 533 e outra da marca Wiso, modelo W904i, foram utilizadas. As voluntárias foram avaliadas no ciclo de intervenção com a mesma balança utilizada no ciclo controle. $\mathrm{O}$ equipamento forneceu, para a pesquisa, o peso e a porcentagem de água corporal.

Os avaliadores foram treinados e atuaram desconhecendo a pesquisa (cegos), bem como seus objetivos. Cada um deles avaliou a mesma voluntária no ciclo controle e intervenção.

As medidas de perimetria foram realizadas em membros superiores, tronco e membros inferiores, com a voluntária em ortostatismo.

Para a análise estatística, foram utilizados os dados de cada segmento do tronco e dos membros e, também, a soma de todas essas medidas, em cada dia de avaliação, nos grupos / ciclos de controle e intervenção.

A média dos valores da perimetria para os segmentos corporais e para o total (soma dos valores da perimetria das voluntárias) do ciclo controle foi comparada com os dados obtidos no ciclo de intervenção.

O peso e a porcentagem de água corporal foram obtidos por meio da balança de bioimpedância com as voluntárias em pé sobre a plataforma metálica da balança.

O peso foi registrado durante os dois ciclos e o peso médio no ciclo de controle foi comparado ao peso médio no ciclo de intervenção.

A porcentagem de água corporal foi verificada nos dois ciclos e a média dessa porcentagem do ciclo controle foi comparada à média do ciclo de intervenção.

Dor abdominal, dor mamária e outros sintomas relatados pela voluntária foram mensurados quantitativamente por meio de um questionário de sintomas, elaborado a partir da adaptação da escala do tipo likert. A média das pontuações do ciclo de controle foi comparada à média das pontuações do ciclo de intervenção.

Em resumo, foram comparadas as médias da perimetria por segmentos corporais e totais, em centímetros; as médias do peso, em quilogramas, a média do percentual de água, em \%, e a média dos escores totais do questionário que investigou a percepção dos sintomas pelos voluntários, nos ciclos controle e intervenção. 
Os dados foram analisados estatisticamente para verificar a importância dos resultados. O programa utilizado foi o SPSS, Statistical Package for the Social Sciences, versão 18.

O Microsoft Office Excel 12.0, 2007 também foi utilizado para apresentar gráficos que mostram a evolução das variáveis peso, porcentagem de água, sintomas e perimetria, comparando os ciclos de controle e intervenção.

Os direitos contidos na Declaração de Helsink II da Associação Médica Mundial foram respeitados. O estudo foi avaliado pelo Comitê de Ética do Instituto Universitário Italiano de Rosário (IUNIR). Também pelo Comitê de Ética da Universidade Newton Paiva, no Brasil, (Parecer CEP - Comitê de Ética e Pesquisa: 329.362 e CONEP - Comissão Nacional de Ética e Pesquisa: 370.289$)$.

\section{RESULTADOS E DISCUSSÃO}

A comparação das médias de todas as variáveis foi realizada considerando os mesmos dias do ciclo controle (sem intervenção) e intervenção (com DLM).

Para todas as variáveis, observou-se que houve redução da média (gráficos 1, 2, 3 e 4). As diferenças nas médias de todas as variáveis (peso, porcentagem de água, perimetria e sintomas relatados) foram positivas e estatisticamente significativas, o que indica que houve redução das médias devido à intervenção. Essa diferença só apresentou valores negativos nas comparações das médias de peso, porcentagem de água e perimetria do $24^{\circ}$ dia. Esse resultado era esperado, visto que o $24^{\circ}$ dia era o primeiro dia do ciclo de intervenção e a intervenção (DLM) foi aplicada após a coleta dos dados das variáveis.

Quando analisada a diferença nas médias das variáveis peso, porcentagem de água, perimetria e sintomas relatados, ficaram evidentes as reduções significativas dessas variáveis no ciclo de intervenção em quase todos os casos. Essa diminuição é atribuída aos efeitos da DLM. O Gráfico 1 apresenta a evolução das médias de peso em cada dia do ciclo de controle e no ciclo de intervenção, em que foi aplicado a DLM. 
Gráfico 1: Evolução das médias de peso nos dias do ciclo controle e intervenção.

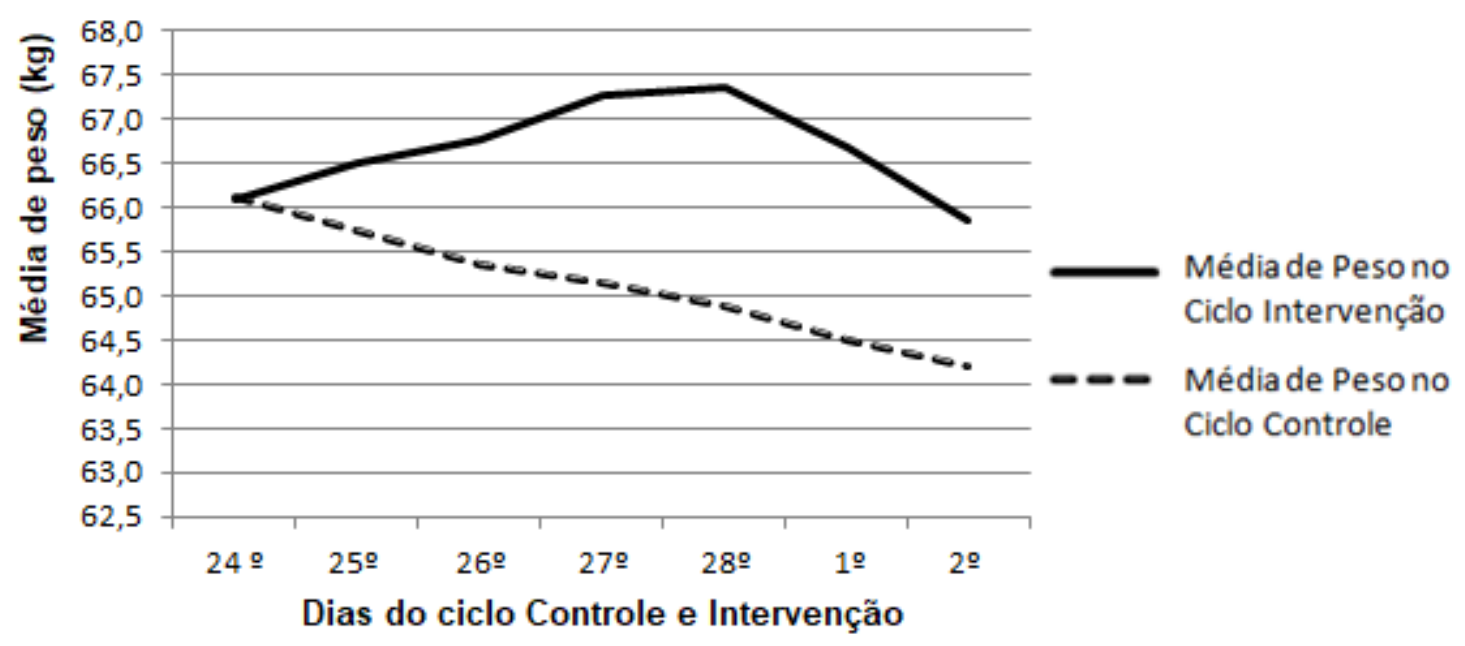

Fonte: Elaborado pelos autores.

Os dados foram coletados do $24^{\circ}$ dia ao $2^{\circ}$ dia do ciclo, ou seja, na fase lútea, porém, sabe-se que as alterações nos hormônios começam por volta do $14^{\circ}$ dia do ciclo, ou seja, no $24^{\circ}$ dia do ciclo as voluntárias já apresentavam, em graus diversos, alterações devido à fase lútea do período pré-menstrual (BERNE; LEVY, 2000).

Essa informação justifica a aparente diminuição da variável peso ao final das medidas no ciclo de intervenção. O que houve foi um retorno ao estado fisiológico da paciente, e não uma alteração acima do normal ou emagrecimento, pois no primeiro dia de avaliação a voluntária provavelmente não estava com o peso normal.

No gráfico pode-se observar que as médias de peso no ciclo de intervenção são menores do que no ciclo de controle. No dia 24 do ciclo, $p=0,634$, nos demais dias, $\mathrm{p}<0,05$.

A evolução das médias da porcentagem de água em cada dia do ciclo de controlo e no ciclo de intervenção estão apresentadas no gráfico 2. 
Gráfico 2: Evolução das médias da porcentagem de água nos dias do ciclo de controle e intervenção.

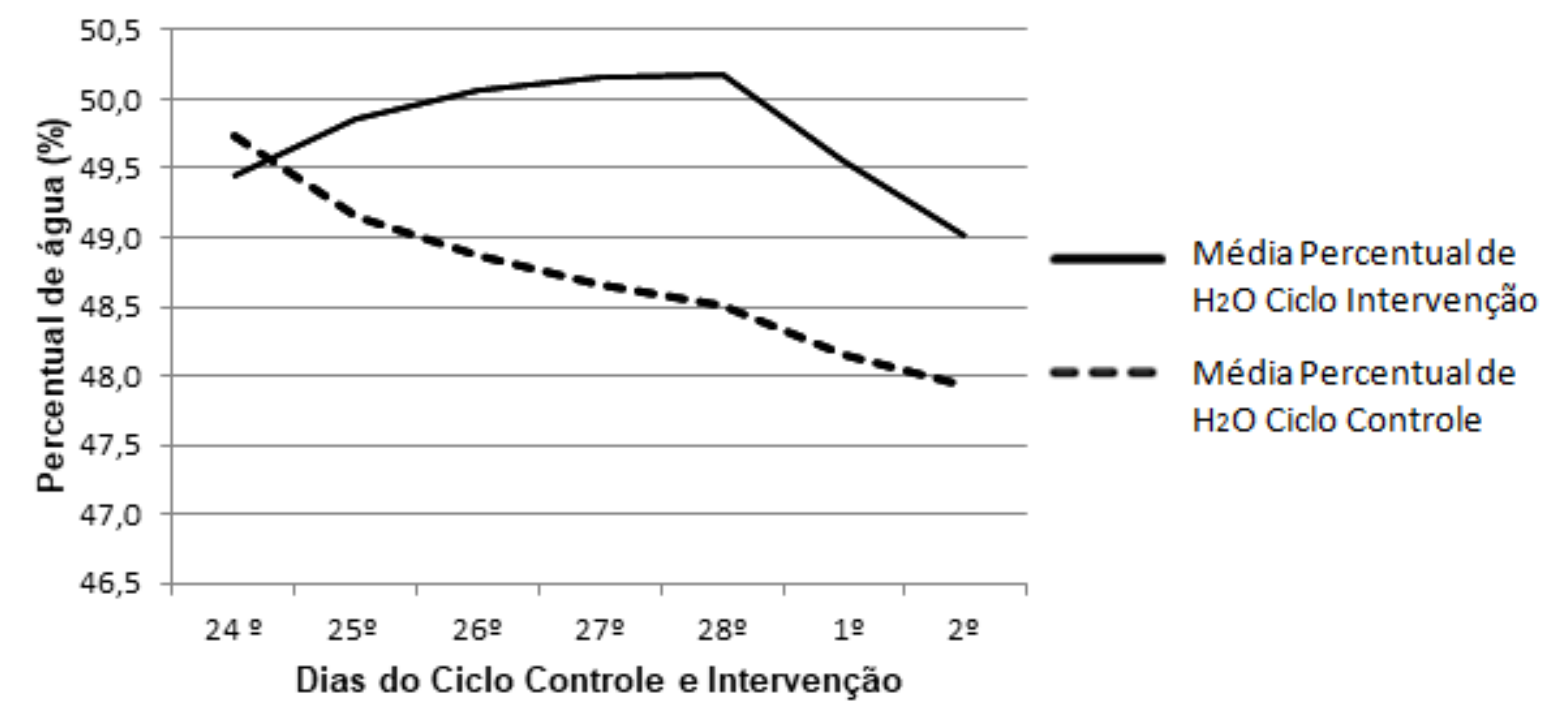

Fonte: Elaborado pelos autores.

As médias do percentual de água são significativamente menores após a intervenção e por causa dela. Essa diferença entre o valor médio foi positiva, o que indica que houve redução nas médias da variável devido à intervenção. A exceção do $24^{\circ}$ dia pode ser explicada porque a voluntária ainda não havia sido submetida a nenhuma sessão de DLM.

O valor de $\mathrm{p}$ foi de 0,008 , ou seja, as diferenças foram significativas para a variável percentual de água, com exceção do $24^{\circ}$ dia de comparação dos ciclos, cujo p foi de 0,368 , pelos mesmos motivos já citados.

O Gráfico 3 mostra a evolução das médias dos valores da perimetria em cada dia do ciclo de controle e no ciclo de intervenção (com a aplicação do DLM). 
Gráfico 3: Evolução das médias da perimetria nos dias do ciclo controle e intervenção.

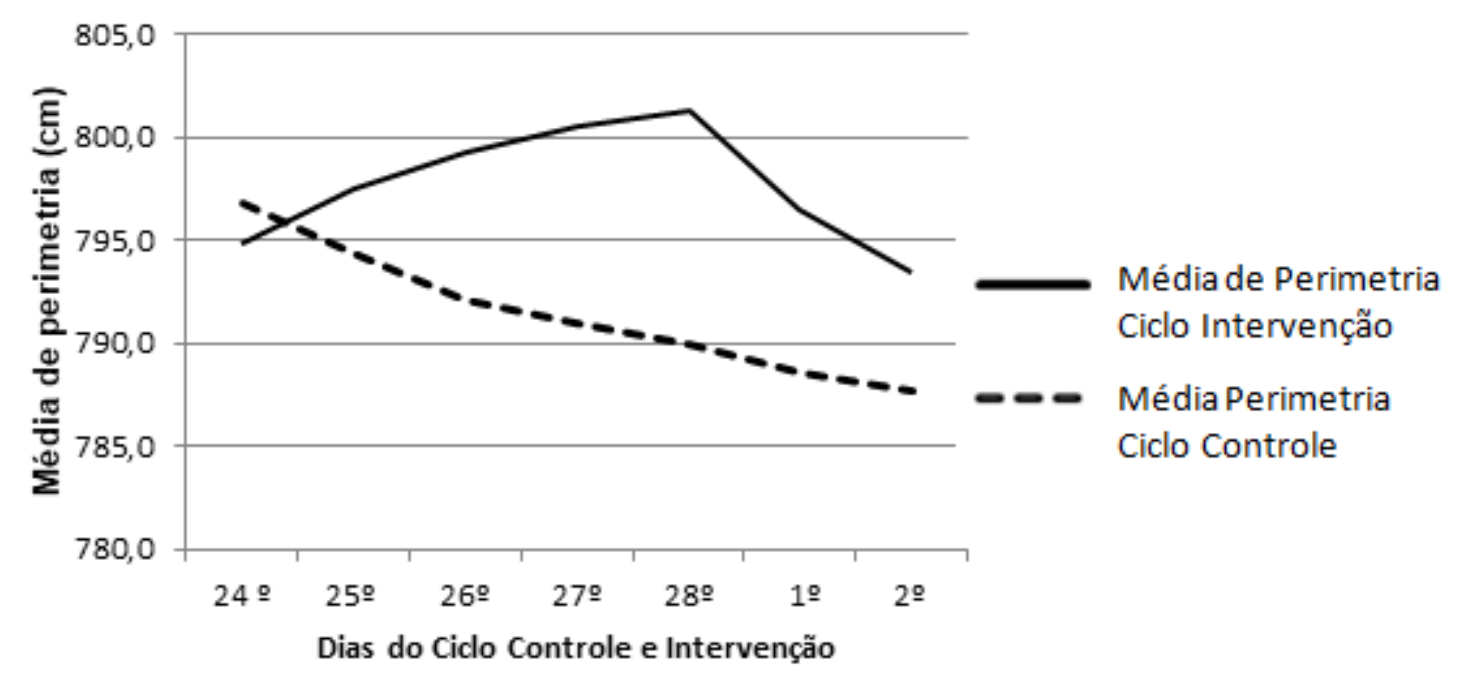

Fonte: Elaborado pelos autores.

A diferença nas médias da perimetria indica que houve redução nas médias dessa variável devido à intervenção. No dia 24 , que foi o primeiro dia de aferição, as medidas foram feitas antes da intervenção, conforme apresentado acima.

$\mathrm{O}$ valor de $\mathrm{p}=0,017$, a partir do $25^{\circ}$ dia, representa que houve redução significativa na média da perimetria. Os motivos da exceção do $24^{\circ}$ dia já foram esclarecidos. Além disso, a diferença nas médias da perimetria foi quase sempre positiva, iniciou em $3,03 \mathrm{~cm}$ e atingiu seu pico no $28^{\circ}$ dia do ciclo, quando atingiu quase 11,4 centímetros.

A partir dos dados obtidos, observou-se que as diferenças nas médias dos valores da perimetria foram evidentes e significativas tanto no tronco quanto nos membros inferiores e superiores, dado este diferente dos resultados obtidos na literatura até o momento.

Ferreira et al. (2010) aplicaram a técnica MLD apenas no abdômen e mamas de suas 4 voluntárias; Nogueira e Silva (2000) verificaram, com n = 10, a maior prevalência de sintomas também nessas regiões. Porém, neste estudo, observaram-se resultados perimétricos no ciclo de intervenção estatisticamente inferiores aos do ciclo de controle, e que todos os segmentos corporais se beneficiaram da DLM.

Essa observação pode ser explicada pela própria anatomia e fisiologia do sistema linfático, segundo Berne e Levy (2000) que afirmam que existe, para a linfa coletada nos tecidos, apenas um caminho a seguir, em direção às veias subclávias; a maior carga de drenagem, gerada pelo tronco, no período menstrual, causa estase ou congestão, afetando a 
drenagem das extremidades superiores e inferiores do corpo.

O Gráfico 4 mostra as médias dos sintomas relatados pelas voluntárias em cada dia do ciclo controle e no ciclo de intervenção, em que foi aplicada a DLM.

Neste gráfico, pode-se observar que as médias das pontuações dos sintomas no ciclo de intervenção são muito mais baixas do que no ciclo de controle. Esses dados mostram o efeito da aplicação da técnica DLM na percepção dos sintomas pela voluntária.

Gráfico 4: Evolução das médias dos sintomas relatados pelas voluntárias nos dias do ciclo controle e intervenção.

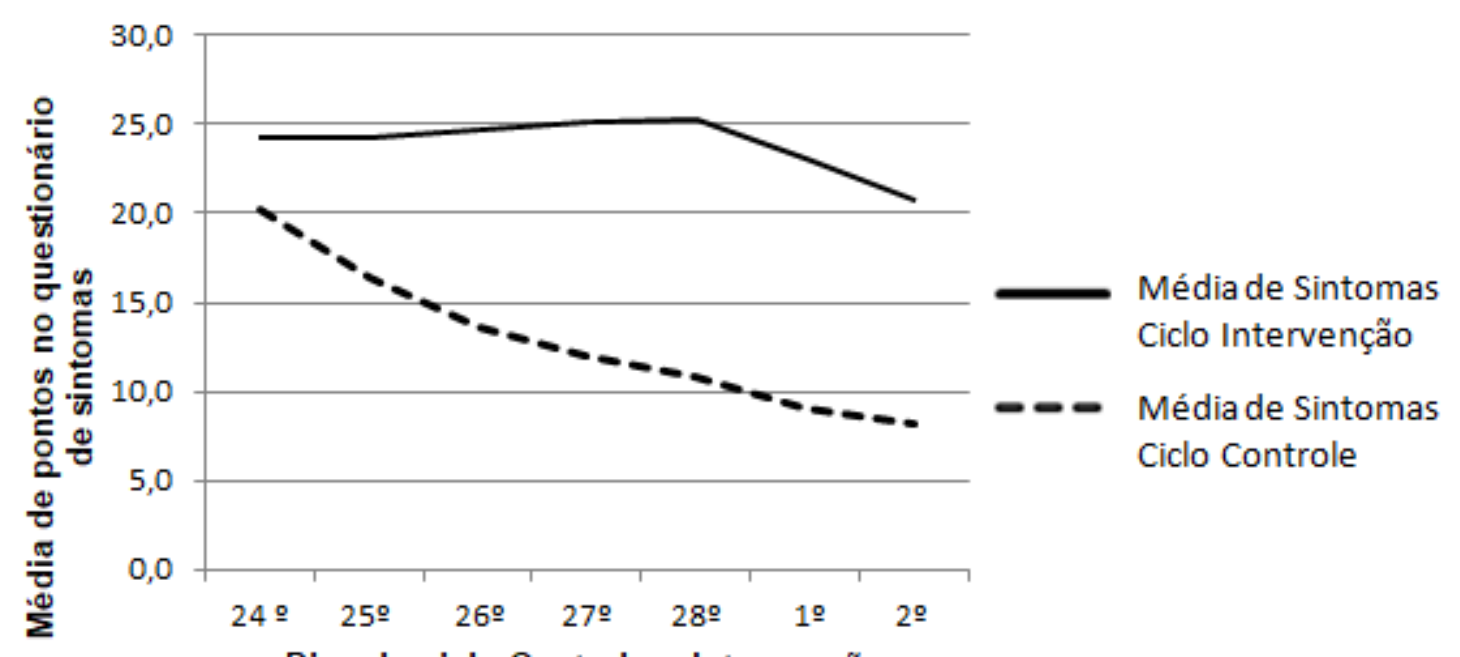

Dias do ciclo Controle e Intervenção

Fonte: Elaborado pelos autores.

Nesse caso, embora os dados tenham sido transformados em dados quantitativos, por meio da pesquisa do tipo Likert, pode-se perceber a influência da subjetividade nos resultados. Somente por conhecerem os objetivos da pesquisa, uma vez que as voluntárias não eram 'cegas', leram o termo de consentimento livre e esclarecido que assinaram, elas apresentaram resultados positivos com a técnica DLM antes mesmo de serem submetidas a ela.

Isso justifica o fato de que, na análise das demais variáveis, o $24^{\circ}$ dia não apresentou diferenças enquanto, no caso da variável percepção dos sintomas pelas mulheres, houve diferenças até mesmo neste dia, ou seja, antes de qualquer aplicação da DLM. Na diferença das médias dos sintomas, houve, em todos os casos, redução significativa dos escores devido à ação da intervenção $(\mathrm{p}<0,05)$. 
Existem divergências na literatura sobre os efeitos da DLM na qualidade de vida das mulheres. Entretanto, a redução da percepção dos sintomas característicos do período prémenstrual, relatados pelas voluntárias, observada neste estudo, influenciou positivamente a qualidade de vida das mulheres.

Em relação a esse dado, Lopes (2002) corrobora ao afirmar que há um efeito calmante e relaxante da DLM, por meio da ativação do sistema nervoso parassimpático, devido à lentidão, suavidade, monotonia e repetição da técnica. Borges (2006) também afirma que a DLM pode trazer benefícios para condições como ansiedade, irritabilidade, depressão, tensão muscular, modificações na dieta, síndrome pré-menstrual, entre outras, devido ao seu efeito no sistema nervoso autônomo.

A monotonia da técnica DLM leva a efeitos relaxantes por influenciar a liberação de substâncias simpatolíticas no corpo (WITTLINGER; WITTLINGER, 1998).

Sousa et al. (2009) em estudo com quatro voluntárias, encontraram resultados na mesma direção. Nas voluntárias, observou-se que a DLM reduziu a gravidade dos sintomas físicos e psicológicos relacionados à retenção de líquidos no período pré-menstrual. Nesse caso, é importante ressaltar que houve redução nos valores das variáveis durante o ciclo de intervenção, mas estatisticamente essas reduções não foram significantes, ou seja, apresentaram p> 0,05.

Ferreira et al. (2010) não encontraram mudanças na qualidade de vida das mulheres em seu estudo. Embora tenham verificado que o DLM foi eficaz no tratamento do edema prémenstrual, pois após a aplicação do DLM, em seu grupo de quatro voluntárias, os principais sintomas: fadiga, inchaço abdominal e mamário, dor pélvica e sensibilidade mamária, foram reduzidos.

Nos estudos com resultados sem relevância estatística para melhoria da qualidade de vida das mulheres, tal situação pode se dever ao pequeno tamanho amostral, que foi de quatro voluntárias para Sousa et al. (2009) e também para Ferreira et al. (2010).

Ainda em relação aos efeitos da DLM na qualidade de vida, Parry (1997) mostrou em sua revisão que estudos com modalidades terapêuticas, como anticoncepcionais orais, vitamina B6, bromocriptina, inibidores da monoamina oxidase, progestogênios sintéticos, espironolactona e progesterona não chegaram a nenhum benefício específico superior ao placebo.

Atualmente, existem ensaios clínicos avaliando a eficácia dos inibidores da recaptação da serotonina para SPM e tem mostrado benefício (WYATT, 2003), geralmente, a taxa de 
resposta é de $60-90 \%$ para o tratamento com drogas versus 30-40\% com placebo (YONKERS; CLARK; TRIVEDI, 1997).

Em um estudo com 3.518 mulheres, 20\% apresentavam sintomas graves de SPM associados à depressão e não foi possível deduzir se a piora dos sintomas foi devido à depressão ou se os sintomas foram agravados pela SPM, ou seja, a síndrome afeta às mulheres fisicamente e psicologicamente. A irritabilidade foi e é descrita como um dos sintomas mais proeminentes em mulheres durante o período pré-menstrual (KNAUSS; STUTZ; WEISS, 2011).

Deve-se considerar que o componente psicológico influencia muito a percepção dos sintomas. Isso pode ser verificado quando se comparam os primeiros dias do ciclo de controle com os primeiros dias do ciclo de intervenção: as variáveis não apresentam alteração significativa, pois não houve intervenção prévia à mensuração. Porém, no caso da variável sintomas relatados pelas voluntárias, mesmo sem aplicação de qualquer intervenção, que caracteriza o momento da coleta de dados no $24^{\circ}$ dia do ciclo de intervenção, os resultados da amostra já estavam significativamente reduzidos.

A presença de irritabilidade e / ou nervosismo foi relatada, de forma importante, por quase $40 \%$ das mulheres pesquisadas por Nogueira e Silva (2000) em Campinas, Brasil.

Um estudo sueco de Andersch et al. (1986) apresentou uma incidência de irritabilidade pré-menstrual moderada em $31 \%$ e severa em $3 \%$ das mulheres.

Em um estudo com 3.027 mulheres francesas, Potter et al. (2009) observaram que apenas $12,2 \%$ queixavam-se de sintomas pré-menstruais moderados a graves.

Há um aumento de peso registrado na balança e a referência subjetiva de que as roupas ficam mais justas. Esse aumento de peso e perimetria, seja real ou apenas uma sensação (ou impressão), causa desconforto, baixa autoestima e pode levar a outros distúrbios, como irritabilidade.

Em suma, embora existam divergências na literatura, em relação aos efeitos da drenagem na qualidade de vida das mulheres, neste estudo houve uma redução significativa nos escores dos sintomas relatados pelas voluntárias, o que contribui positivamente para a qualidade de vida das mulheres.

Além disso, a diferença nas médias de todas as variáveis aumentou gradativamente e atingiu seu pico no $28^{\circ}$ dia do ciclo. Esse dado é consistente com a distribuição dos sintomas na fase lútea, pois os sintomas são cíclicos e aumentam gradativamente, com pico geralmente 
neste dia, provavelmente ocorrendo no ciclo controle. Porém, no ciclo de intervenção, a técnica DLM evitou os aumentos das variáveis, comprovando a eficácia da DLM.

A redução das médias de peso, porcentagem de água e perimetria estão de acordo com os dados obtidos na literatura (MACEDO et al., 2006; FERREIRA et al., 2010; SOUSA et al., 2009; SIMÕES; FERNANDES, 2007).

Macedo et al. (2006) concluíram que a DLM é um recurso eficaz para o tratamento do edema pré-menstrual, pois pode promover a redistribuição de água, eletrólitos e hormônios no sistema vascular. Esses autores avaliaram a composição corporal: o percentual de hidratação, gordura corporal, circunferência da cintura; e substâncias eliminadas na urina: densidade e pH em dez voluntárias, no período pré-menstrual antes e após a aplicação da DLM. Com isso, identificaram, após o processo de DLM, alterações significativas na circunferência da cintura ( $\mathrm{p}=0,01$ ), bem como aumento do $\mathrm{pH}$ e diminuição da densidade da urina das voluntárias.

Ferreira et al. (2010) corroboram com este estudo, uma vez que seus resultados mostram efeitos benéficos da DLM na redução do edema pré-menstrual nas quatro mulheres estudadas. Eles obtiveram diminuição estatisticamente significativa nos perímetros do apêndice xifóide e umbilical, embora com número reduzido de voluntárias.

Também Sousa et al. (2009) em estudo com quatro voluntárias, encontraram resultados na mesma direção. Nas voluntárias, observou-se que a DLM reduziu o peso corporal, a porcentagem de água corporal e a perimetria corporal no ciclo de intervenção, mas estatisticamente essas reduções tiveram $\mathrm{p}>0,05$, provavelmente devido ao pequeno tamanho da amostra.

Simões e Fernandes (2007) mediram o volume urinário de cinco voluntárias, após a aplicação da técnica de DLM, no dia 10 de dois ciclos menstruais consecutivos, e constataram que houve aumento do volume urinário em todas as mulheres no segundo mês em relação ao primeiro. Esses dados reforçam os deste trabalho em que houve redução no percentual de água corporal ao se comparar o ciclo de intervenção com o ciclo controle.

Embora não sejam encontrados mais estudos relacionando SPM com DLM, Ballone (2005) diz que os sintomas físicos, principalmente, estão relacionados à característica de retenção de água do período pré-menstrual, e que a inflamação pode ser responsável pela sensibilidade em nas mamas, para dores abdominais e musculares e nas mãos e pés. Para Borges (2006), o ácido lático proveniente de reações químicas em músculos fatigados, que causa dores 
e espasmos musculares, pode ser eliminado mais rapidamente do organismo por meio de DLM, reduzindo a dor muscular e a inatividade.

Mudanças na produção de serotonina e GABA (ácido gama-aminobutírico) nas regiões da hipófise e amígdala e outras regiões do cérebro também alteram a concentração de prolactina que justifica a presença de cefaleia (YONKERS; CLARK; TRIVEDI, 1997).

Ribeiro, Hardy e Hebling (2007) avaliaram um grupo de 420 mulheres em Campinas, $\mathrm{SP}$, com idades entre 25 e 34 anos. Seus resultados mostram que $18,1 \%$ das mulheres tiveram dois dias de duração da dor, $55 \%$ apresentaram desconforto de forte intensidade e $36,2 \%$ relataram a interferência da dor ou desconforto em seus estudos, trabalho e outras atividades de rotina. Afirmaram, ainda, que dor ou inchaço nos seios, abdômen e pernas estão intimamente relacionados ao excesso de líquido intersticial que causa distensão dos tecidos e, dependendo da região afetada, pode contribuir para o agravamento dos sintomas.

$\mathrm{Na}$ revisão de Yonkers, O'Brien e Eriksson (2008), muitos regimes de tratamento considerados eficazes para a SPM também são apresentados, mas poucos são apoiados por evidências clínicas. Os tratamentos não são necessariamente iguais para todos os sintomas. A avaliação da melhoria de algum sintoma, por meio de levantamento ou escala, pode obscurecer o efeito específico sobre outro sintoma. Esses autores concluem que o tratamento deve ser individualizado de acordo com o sintoma.

Na população deste estudo, um dos critérios de inclusão foi o sintoma de retenção hídrica ou edema pré-menstrual e pôde-se verificar que houve redução do edema, evidenciado pela redução do percentual de água e valores de perimetria no ciclo intervenção, se comparados ao controle, e também o peso, que neste caso pode ser associado à retenção de líquidos.

Na mesma mulher os sintomas podem não ser os mesmos e sua intensidade pode variar. Também podem ser cognitivos, comportamentais, somáticos e neurovegetativos, como labilidade emocional, diminuição da atividade, autoestima, preferência por determinados alimentos, entre outros (NOGUEIRA; SILVA, 2000).

Carvalho et al. (2009) realizaram um estudo com 259 mulheres universitárias, por meio de um questionário sobre sintomas menstruais, cujos sintomas físicos mais evidentes foram: peso associado a desconforto e dor abdominal (25,5\%), dismenorreia (22,8\%), 26,3\% tinham sensação de inchaço e $26,6 \%$ tinham seios mais sensíveis e inchados, além de outros sintomas somáticos e emocionais da SPM. Isso mostra que o edema está presente em mais de $50 \%$ das mulheres neste estudo. 
O edema pode ser explicado por alterações na concentração dos hormônios LH e FSH durante a fase lútea tardia, que coincide com o período das mulheres com SPM, associada a níveis elevados de aldosterona; além de mudanças nas concentrações de renina e aumento dos níveis de progesterona (ROSENFELD et al., 2008). Esse dado explica porque o $28^{\circ}$ dia corresponde ao pico das alterações nas variáveis deste estudo no ciclo de controle.

Houve pico de sintomatologia no $28^{\circ}$ dia em todas as variáveis deste estudo, e isso é esperado porque esse dia corresponde à fase lútea tardia. Assim, fica esclarecido porque as médias das variáveis peso, porcentagem de água, perimetria e sintomas relatados tiveram o ponto alto de seus sintomas no $28^{\circ}$ dia do ciclo controle.

Ao contrário, no ciclo de intervenção, não houve tal pico de sintomas devido ao efeito da DLM na eliminação dos fluidos acumulados.

Solicitou-se das voluntárias ingerir alimentos e líquidos no ciclo de intervenção, de maneira semelhante ao efetuado por elas no ciclo controle, conforme registros no diário nutricional, de forma a não promover mudanças nas variáveis estudadas em decorrência de alterações alimentares. Isso porque a alteração do apetite é um sintoma físico presente no período pré-menstrual, há preferência por alimentos altamente calóricos, desejo por doces e aumento do apetite em geral (BALLONE, 2005). Não houve restrição alimentar para as voluntárias, apenas solicitou-se delas, seguir a mesma rotina do ciclo controle no ciclo de intervenção.

Neste trabalho, observou-se que o ganho de peso caracteriza o período pré-menstrual, provavelmente devido à ingestão alimentar e à presença de edema. O edema causa não apenas ganho de peso, mas também aumento das circunferências corporais e, portanto, dos valores da perimetria. Como a ingestão alimentar foi controlada de modo a não ser diferente nos ciclos controle e intervenção, e não houve voluntárias que relataram não seguir a mesma ingestão alimentar e hídrica do controle, as diferenças de peso podem ser atribuídas à retenção de líquidos.

Embora poucos estudos relacionem a SPM à DLM, existem dados que comprovam a eficácia da drenagem para o edema, portanto, se houver clareza quanto à relação do edema com os sintomas pré-menstruais e quanto ao tratamento direcionado ao sintoma, não se pode negar que a DLM seja uma opção de tratamento para SPM.

Um ponto fraco deste trabalho não foi controlar diretamente a ingestão de sal. As voluntárias apenas foram solicitadas a fazer a mesma alimentação, mas a quantidade de sal que ? 
ingeriram não foi controlada. Em um estudo futuro, seria interessante observar essa questão porque é sabido que as quantidades de sal podem interferir na retenção de líquidos.

\section{CONCLUSÃO}

Concluiu-se que as médias do peso, percentual de água, perimetria e sintomas relatados pelas voluntárias foram significativamente menores, na mesma mulher, após a intervenção, devido ao efeito da DLM.

Pôde-se notar, também, que as variações nas médias da perimetria são significativas no tronco, membros inferiores e membros superiores. Assim, justifica-se aplicar a técnica de DLM em todo corpo com o objetivo de reduzir as variações. Embora a técnica possa ser direcionada para a região do tronco em que as alterações são mais evidentes, por se tratar da localização uterina, na prática clínica, deve-se drenar os membros inferiores e superiores, uma vez que este estudo evidenciou variações nas médias de perimetria de todos os segmentos corporais.

Pode-se dizer que, ao reduzir o edema, a DLM reduz as alterações do peso corporal, da perimetria e da porcentagem de água corporal que são geradas no período pré-menstrual, além de diminuir a percepção, pelas mulheres, dos sintomas que são gerados nesta fase do ciclo menstrual.

Embora a SPM esteja presente no dia a dia de muitas mulheres, existem poucos estudos científicos relacionando a DLM como um tratamento. Portanto, dados baseados em evidências são importantes para implementar a DLM como uma terapêutica alternativa ou complementar à medicação e cirurgia, para reduzir os sintomas causados pela SPM, a fim de melhorar a qualidade de vida, saúde, e relação interpessoal da mulher com a família, no trabalho e na sociedade (SOUSA et al., 2009; FERREIRA et al., 2010).

O uso da DLM em mulheres com SPM apresentou resultados benéficos, ainda que mais estudos sejam necessários.

\section{REFERÊNCIAS}

ANDERSCH B, WENDESTAM C, HAHN L, OEHMAN R. Premenstrual complaints. I. Prevalence of premenstrual symptoms in a Swedish urban population. J Psychosom Obstet Gynaecol 1986; 5:39-49. 
AUGUSTO N, URBANETZ AA. Síndrome de tensão pré-menstrual. I. Aspectos clínicos e fisiopatologia. Femina 1985; 13:682-90.

BALlONE GJ. Tensão pré-menstrual - TPM. Portal da Psiquiatria, Fev. 2005. Disponível em: http://virtualpsy.locaweb.com.br/index.php?art=247\&sec=14. Acesso en 17/04/2008.

BERNE RB, LEVY MN. Tratado De Fisiologia Humana. 4 Ed. Rio de Janeiro: Guanabara Koogan, 2000.

BORGES FS. Modalidades Terapêuticas nas Disfunções Estéticas. São Paulo: Phorte, 2006. 680p.

CAMPAGNE DM, CAMPAGNE G. The Premenstrual Syndrom Revisited. European Journal of Obstetrics \& Gynecology and Reproductive Biology 130 (2007): 4-17.

CARVALHO VCP, CANTILINO A, CARREIRO NMP, SÁ LF, SOUGEY EB. Repercussões do transtorno disfórico pré-menstrual entre universitárias. Revista de Psiquiatria RS.; 31(2):105-111, 2009.

FERREIRA JJ, MACHADO AFP, TACANI R, SALDANHA MES, TACANI PM, LIEBANO RE. Drenagem linfática manual nos sintomas da síndrome pré-menstrual: estudo piloto. Fisioterapia e Pesquisa, São Paulo, v.17, n.1, p.75-80, jan/mar. 2010.

GODOY JMP, GODOY MFG. Drenagem linfática manual: novo conceito. J Vasc Br 2004; Vol. 3, No 1:77-80.

GONDA X, LAZÁRY J, TELEK T, PAP D, KÁTAI Z, BAGDY, G. Mood parameters and severe physical symptoms of the female reproductive cycle. Neuropsychopharmacologia Hungarica 2008, X/2; 83-88.

GUIRRO ECO, GUIRRO RRJ. Fisioterapia dermato-funcional: fundamentos, recursos, patologias. $3^{\text {a }}$ ed. São Paulo: Manole, 2004.

GUYTON AC, HALL JE. Fisiologia Humana e Mecanismos das Doenças. $6^{\mathrm{a}}$ ed. Rio de Janeiro: Guanabara Koogan,1998.

KNAUSS FC, STUTZ ZE, WEISS C. The interrelation between premenstrual, syndrome and major depression: Results from a population-based sample, BMC Public Health, 11:795, 2011.

KWAN I, ONWUDE JL. Premenstrual syndrome. Clin Evid (Online) 2009; 2009: 0806. Published online 2009 December 21.

LEDUC A, LEDUC O. Drenagem Linfática: teoria e Prática. $2^{\mathrm{a}}$ ed. São Paulo: Manole, 2007. 
LOPES MLM. Drenagem linfática manual e a estética. Blumenau: Odorizzi, 2002. 130 p.

MACEDO FBM, COUTINHO EST, SILVA ACA, KHOURI S, LIMA FPS. Efeitos da drenagem linfática manual no edema pré-menstrual. Revista Univap. SP, v.13, n.24, out.2006.

NOGUEIRA CWM, SILVA JLP. Prevalência dos sintomas da síndrome pré-menstrual. Rev Bras Ginecol Obstet. 2000;22(6):347-51.

PARRY BL. Psychobiology of premenstrual dysphoric disorder. Semin Reprod Endocrinol. 1997;15(February (1)):55-68.

POTTER J, BOUYER J, TRUSSELL J, MOREAU C. Premenstrual Syndrome Prevalence and Fluctuation over Time: Results from a French Population-Based Survey. Journal of Women's Health.Volume 18, Number 1: 31-39, 2009.

RIBEIRO CP, HARDY E, HEBLING HM. Preferências de mulheres Brasileiras quanto a mudanças na menstruação. Revista Brasileira de Ginecologia e Obstetrícia. 29(2):74-79, 2007.

ROSENFELD R, LIVNE D, NEVO O, DAYAN L, MILLOUL V, LAVI S, GIRIS J. Hormonal and Volume Dysregulation in Women With Premenstrual Syndrome. American Heart Association. February 7. 2008.

SIMÕES NP, FERNANDES TS. Mensuração do volume urinário após a aplicação da técnica de drenagem linfática manual. Resumo publicado na Revista Fisioterapia Brasil, v. 3, fev, 2007.

SOUSA FC, MELO LG, SARMENTO MS, DURANTE DS, DINIZ, JS. Verificação dos efeitos da Drenagem linfática Manual em mulheres com queixa de retenção hídrica no período pré-menstrual. Revista Kinesia, v. 02, p.-55-62, 2009.

WITTLINGER G, WITTLINGER, H. Textbook of Dr. Vodder's Manual Lymph Drainage. Basic Course. V.1, 7 ed. Thieme, 1998, p.29-40; 80-98.

WYATT KM, DIMMOCK PW, O’Brien PMS. Selective serotonin reuptake inhibitors for premenstrual syndrome. Cochrane Database of Syst Rev. 2003; 3:CD001396.

YONKERS KA, CLARK RH, TRIVEDI MH. The psychopharmacological treatment of nonmajor mood disorders. In: RUSH, AJ. Editor. Mood disorders: systematic medication management-modern problems of pharmacopsychiatry. 2. Basel: Karger; 1997. p. 146-66.

YONKERS KA, O’BRIEN PM, ERIKSSON E. Premenstrual syndrome. NIH Public Access. Lancet. 2008 April 5; 371(9619): 1200-1210. 


\section{Declaração de Interesse}

Os autores declaram não haver nenhum conflito de interesse.

\section{Financiamento}

Financiamento próprio.

\section{Colaboração entre autores}

Sousa idealizou o estudo, participou do planejamento da pesquisa, supervisionou a coleta de dados, realizou análise dos dados, criação de tabelas e figuras, escreveu o manuscrito em Espanhol, bem como a tradução para Língua Portuguesa.

Nascimento colaborou com a revisão do texto, com a análise dos dados $e$ adicionou partes significativas.

Alonso participou do planejamento do estudo, assim como com a revisão da redação da pesquisa em língua estrangeira. 